\title{
DNase I aggravates islet $\beta$-cell apoptosis in type 2 diabetes
}

\author{
BIN ZHU $^{1 *}$, LEI ZHANG ${ }^{2 *}$, YUE-YING ZHANG ${ }^{3}$, LEI WANG $^{4}$, XIN-GANG LI ${ }^{1}$, TENG LIU ${ }^{1}$, \\ YU-KE FU ${ }^{1}$, YAN-FEI ZHENG ${ }^{4}$, PING LI $^{5}$ and ZHI-GANG ZHAO ${ }^{1}$
}

\begin{abstract}
${ }^{1}$ Department of Pharmacy, Beijing Tiantan Hospital, Capital Medical University, Beijing 100050; ${ }^{2}$ Department of Pharmacy, Beijing Shijitan Hospital, Capital Medical University, Beijing 100038; ${ }^{3}$ Department of Chinese Medicine, Beijing Hepingli Hospital, Beijing 100013; ${ }^{4}$ Department of Chinese Medicine, The Third Affiliated Hospital of Beijing University of Chinese Medicine, Beijing 100050; ${ }^{5}$ Department of Nephrology, China-Japan Friendship Hospital, Beijing 100029, P.R. China
\end{abstract}

Received February 27, 2015; Accepted March 7, 2016

DOI: $10.3892 / \mathrm{mmr} .2016 .5102$

\begin{abstract}
Deoxyribonuclease I (DNase I) is an endonuclease responsible for the destruction of chromatin during apoptosis. However, its role in diabetes remains unclear. The aim of the current study was to investigate the role of DNase I combined with high glucose levels in $\beta$-cell apoptosis. Human samples were collected and the DNase I activity was examined. High glucose-cultured INS-1 cells were transfected with DNase I small interfering RNA (siRNA) and the cell apoptosis was examined by western blotting and flow cytometry. Cell viability was analyzed by the Cell Counting Kit- 8 assay. Cell apoptosis resulting from $50 \mathrm{mU} / \mu 1$ DNase I was also observed by flow cytometry, terminal deoxynucleotidyl transferase dUTP nick-end labeling stain and western blotting. Compared with healthy controls, the serum DNase I activity of patients with diabetes was significantly increased $(\mathrm{P}<0.05)$. In addition, DNase I expression was observed to be significantly increased in human pancreatic tissues. The addition of high glucose upregulated the cell apoptotic rate, whereas DNase I knockdown significantly reduced apoptosis in cells treated with high glucose. In addition, the western blotting results indicated that caspase- 3 was increased subsequent to treatment of cells with $30 \mathrm{mM}$ high glucose, however, this increase can be reversed by transfection with DNase I siRNA $(\mathrm{P}<0.05)$. Compared with cells cultured in normal conditions and high glucose, $50 \mathrm{mU} / \mu \mathrm{l}$ DNase I was able to significantly increase the cell apoptotic rate and level of caspase-3. DNase I activity was observed to be
\end{abstract}

Correspondence to: Professor Ping Li, Department of Nephrology, China-Japan Friendship Hospital, 2 Yinghuayuan E Street, Beijing 100029, P.R. China

E-mail:1p867@163.com

Professor Zhi-Gang Zhao, Department of Pharmacy, Beijing Tiantan Hospital, Capital Medical University, 6 Tiantan Xili, Dongcheng, Beijing 100050, P.R. China

E-mail: 1022zzg@sina.com

*Contributed equally

Key words: DNase I, high glucose, $\beta$ cell, apoptosis increased in type 2 diabetes, and high glucose combined with increased DNase I is suggested to aggravate $\beta$-cell apoptosis.

\section{Introduction}

$\beta$-cell dysfunction is a key characteristic in the pathogenesis of type 2 diabetes $(1,2)$ and hyperglycemia serves a direct role in pancreatic $\beta$-cell dysfunction and death (3). However, the mechanism underlying glucose-induced $\beta$ cell apoptosis and dysfunction remain to be fully understood. Deoxyribonuclease I (DNase I) is a $38-\mathrm{kDa}$ glycoprotein that is a type of $\mathrm{Ca}^{2+} / \mathrm{Mg}^{2+}$-dependent non-restriction nuclease, which can hydrolyze phosphodiester bonds in single and double stranded DNA (4). Thus, endogenous DNase I has been suggested as a candidate endonuclease facilitating chromatin breakdown during apoptosis.

As a secretory endonuclease, DNase I is the predominant nuclease observed in body fluids such as serum and urine. In mammals, the pancreas exhibits the highest DNase I activity (5), with 60-65\% total serum DNase I secreted by the pancreas (6). Previously, two diseases, systemic lupus erythematosus (SLE) and acute myocardial infarction (AMI), have been demonstrated to be associated with alterations in serum levels of DNase I $(7,8)$. The association between DNase I and type 2 diabetes remains to be fully elucidated.

Given the essential role of pancreatic tissue in the development of metabolic syndrome, it is suggested that there may be a functional significance of DNase I in the morbidity of type 2 diabetes (9). In the present study, DNase I activity in patients with type 2 diabetes was assessed, with the aim to explore the role of DNase I in high glucose conditions in association with $\beta$ cell apoptosis.

\section{Materials and methods}

Human serum collection. Individuals who were diagnosed with type 2 diabetes [glycated hemoglobin (HbAlc) $\geq 6.5 \%$ or fasting blood glucose $\geq 7.0 \mathrm{mmol} / 1 ; 35-65$ years old] were recruited at the China-Japan Friendship Hospital (Beijing) between September and December, 2012 (Table I). Patients who were pregnant, or presented with other acute or chronic complications, including malignant tumors, SLE, AMI, uncontrolled hypertension or other serious illnesses were 
excluded from the study. Healthy volunteers with normal glucose, triglyceride and cholesterol levels and with the absence of other diseases were enrolled in the study. A consent form was signed by all subjects prior to enrollment. Blood samples were taken from the antecubital vein without any anticoagulant, and were centrifuged for $10 \mathrm{~min}$ at $1,500 \mathrm{x} \mathrm{g}$ at an ambient temperature. Serum samples were aliquoted into $200 \mu \mathrm{l}$ samples, which were stored at $-80^{\circ} \mathrm{C}$ until required for further experiments. The concentration of calcium, blood glucose and HbAlc in the serum was measured using the Hitachi 7600 Auto-Biochemistry Instrument (Hitachi, Ltd., Tokyo, Japan) with the reagents and methods provided by the manufacturer. This study was approved by the ethics committee of the China-Japan Friendship Hospital (Beijing, China).

Human tissues. Surgically removed human pancreatic cancer tissue specimens were obtained with approval from the China-Japan Friendship Hospital Institutional Review Board (Beijing, China). Human pancreatic tissues were collected from patients with pancreatic cancer with a history of diabetes for at least 4 years ( 3 male and one female) or without diabetes as control ( 2 male and 2 female). The patients were aged from 50 to 65 . Written informed consent was obtained from each patient. Surgically removed pancreatic tissues from patients with pancreas cancer, with or without diabetes, were used for the observation of DNase I. Sections of tumor-free pancreas were immediately placed into ice-cold fixative [ $4 \%$ formaldehyde $+0.1 \%$ glutaraldehyde in phosphate-buffered saline (PBS)] subsequent to resection, then were processed for immunohistochemistry.

Measurement of DNase I activity. DNase I activity was measured using the radial enzyme-diffusion method. Briefly, calf thymus DNA (Sigma-Aldrich, St. Louis, MO, USA) was used as the substrate and was mixed with SYBR Green I (Unique, Beijing, China) in DNase buffer (Worthington Biochemical Corporation, Lakewood, NJ, USA). The end concentration of substrate DNA in the mixture was 500 ng/ml. Regular (2\%) agarose (Biowest, HongKong, China) was melted using distilled water and mixed with the solution. The mixed gel was then poured into the 96-well microplate. The serum samples $(2 \mu \mathrm{l})$ were then injected into the center of each well of the gel plate. A total of $2 \mu \mathrm{l}$ DNase I (Worthington Biochemical Corporation) at concentrations of $0.034-1.1 \mathrm{U} / \mathrm{ml}$ were additionally added into the gel plate to calculate the standard curve. Each sample was performed in duplicate. The gel plate was then incubated at $37^{\circ} \mathrm{C}$ in a airtight black box for $12 \mathrm{~h}$. The areas of hydrolyzed DNA were assessed with a gel documentation and image analysis system (ChampGel 5500; Beijing Sage Creation Science Co., Ltd., Beijing, China) and were quantitated with Image-Pro Plus software, version 6.0 (Media Cybernetics, Inc., Rockville, MD, USA).

Immunohistochemistry for insulin, glucagon and DNase I. Immunohistochemistry was conducted in $2 \mu \mathrm{m}$-thick paraffin (Sinopharm Chemical Reagent, Beijing, China)-embedded pancreatic sections mounted in polylysine-coated slides. Unstained sections were deparaffinized and rehydrated in xylene (Sinopharm Chemical Reagent) and graded ethanol. Subsequent to rinsing in distilled water, endogenous peroxidase was blocked with 3\% hydrogen peroxide (Sinopharm Chemical Reagent) for 30 min to reduce nonspecific binding, then were incubated with primary antibodies, mouse anti-insulin (1:5,000; ZM-0155; OriGene Technologies, Inc., Beijing, China), rabbit anti-glucagon (1:5,000; ZA-0119; OriGene Technologies) and rabbit anti-DNase I (1:1,000; sc-30058; Santa Cruz Biotechnology, Inc., Dallas, TX, USA). The primary antibodies were detected using the anti-mouse/rabbit Poly-Horseradish-Peroxidase from the Immunohistochemistry Ready-to-use Detection kit (GTVision ${ }^{\mathrm{TM}}$ III Detection System/Mo\&Rb; Gene Tech Biotechnology Co., Ltd., Shanghai, China). Visualization was performed using diaminobenzidine chromogen buffer. Sections were counterstained with hematoxylin (Sinopharm Chemical Reagent). Digital morphometric analyses were performed using the Leica DM5000 optical microscope with Leica Qwin Plus analysis software DM5000 (Leica Microsystems, Inc., Buffalo Grove, IL, USA).

INS-1 cell culture. The insulin-secreting cell line INS-1 (Cell Resource Center of Peking Union Medical College, Beijing, China) was cultured in RPMI-1640 (Hyclone, Logan, UT, USA), supplemented with $10 \%$ fetal calf serum (FCS; Gibco; Thermo Fisher Scientific, Inc., Waltham, MA, USA), $2 \mathrm{mmol} / 1$ L-glutamine, $10 \mathrm{mmol} / 1 \mathrm{HEPES}, 1 \mathrm{mmol} / 1$ sodium pyruvate and $50 \mathrm{mmol} / 1$ 1,2-mercaptoethanol (all from Sigma-Aldrich) in a humidified atmosphere $\left(5 \% \mathrm{CO}_{2}\right.$, $\left.37^{\circ} \mathrm{C}\right)$.

Small interfering RNA (siRNA) transfection. DNase I siRNAs were synthesized by Shanghai GenePharma Co., Ltd. (Shanghai, China). The siRNA sequences were as follows: DNase I, 5'-GCCGCAAAAGCUACAAGGATT-3' and 5'-UCCUUGUAGCUUUUGCGGCTT-3'; negative control siRNA, 5'-UUCUCCGAACGUGUCACGUTT-3' and 5'-ACG UGACACGUUCGGAGAATT-3'. INS-1 cells were cultured in Opti-MEM (Invitrogen; Thermo Fisher Scientific, Inc.) for $12 \mathrm{~h}$ and then transfected with the siRNAs and Lipofectamine $^{\mathrm{TM}} 2000$ (Invitrogen; Thermo Fisher Scientific, Inc.). The cells were then plated into wells and incubated at $37^{\circ} \mathrm{C}$ in a $\mathrm{CO}_{2}$ incubator. A total of $6 \mathrm{~h}$ later, the medium was replaced with RPMI-1640 containing either an $11.1 \mathrm{mM}$ or $30 \mathrm{mM}$ concentration of glucose (Sinopharm Chemical Reagent). All experiments using siRNA-transfected INS-1 cells were performed $48 \mathrm{~h}$ subsequent to transfection unless otherwise stated.

RNA extraction and reverse transcription-quantitative polymerase chain reaction $(R T-q P C R)$. Total RNA $(2 \mu \mathrm{g})$ was converted to first-strand cDNA using the RevertAid ${ }^{\mathrm{TM}}$ First Strand cDNA Synthesis kit (Fermentas; Thermo Fisher Scientific, Inc.). RT-qPCR analysis was used to measure the relative levels of DNase I, Bcl-2 and caspase-3 mRNA expression. The amplification was performed on an ABI 7500 thermocycler (Applied Biosystems; Thermo Fisher Scientific, Inc.). The nucleotide sequences of the primers were as follows: DNase I, forward 5'-GGTCCGAGAGTTTGC GATTGT-3' and reverse 5'-TGCAGCCAGCATTGAAAT CTC-3'; caspase-3, forward 5'-AGCAGTTACAAAATGGAT 
Table I. Baseline patient demographic and background characteristics.

\begin{tabular}{lcc}
\hline Characteristic & Healthy controls & Diabetes patients \\
\hline $\mathrm{N}$ (male/female) & $51(26 / 25)$ & $66(44 / 22)$ \\
Age (years) & $50.47 \pm 8.37$ & $52.68 \pm 8.91$ \\
FPG (mmol/l) & $5.07 \pm 0.37$ & $8.07 \pm 3.07^{\mathrm{a}}$ \\
$\mathrm{HbA1C}(\%)$ & $5.5 \pm 0.35$ & $8.77 \pm 1.63^{\mathrm{b}}$ \\
Systolic pressure (mmHg) & $118.47 \pm 17.49$ & $130.45 \pm 13.22^{\mathrm{b}}$ \\
Diastolic pressure $(\mathrm{mmHg})$ & $77.93 \pm 12.25$ & $79.68 \pm 7.71$ \\
BMI $\left(\mathrm{kg} / \mathrm{m}^{2}\right.$ ) & $23.24 \pm 2.91$ & $26.83 \pm 3.73$ \\
Cholesterol (mmol/l) & $4.38 \pm 0.58$ & $4.75 \pm 1.18$ \\
Triglyceride (mmol/l) & $1.11 \pm 0.43$ & $1.81 \pm 0.97^{\mathrm{b}}$ \\
High density lipoprotein $(\mathrm{mmol} / \mathrm{l})$ & $1.55 \pm 0.32$ & $1.23 \pm 0.32^{\mathrm{b}}$ \\
Low density lipoprotein $(\mathrm{mmol} / \mathrm{l})$ & $2.64 \pm 0.42$ & $2.64 \pm 0.81$
\end{tabular}

Values are presented as the mean \pm standard deviation, unless indicated otherwise. FPG, fasting plasma glucose level; HbAlc, hemoglobin Alc; BMI, body mass index; ${ }^{a} \mathrm{P}<0.01$ and ${ }^{b} \mathrm{P}<0.001$ vs. the healthy control.

TAC-3' and reverse 5'-ATCTCCATGACTTAGAATCAC-3'; Bcl-2, forward 5'-TGGTGGACAACATCGCTCTGT-3' and reverse 5'-CCCAGGTATGCACCCAGAGTG-3'; $\beta$-actin, forward 5'-ATCGTGCGTGACATTAAGGAGAAG-3' and reverse 5'-AGGAAGGAAGGCTGGAAGAGTG-3'. The PCR reaction conditions were as follows: $94^{\circ} \mathrm{C}$ for $1 \mathrm{~min}$ followed $56^{\circ} \mathrm{C}$ for $30 \mathrm{sec}$ and $72^{\circ} \mathrm{C}$ for 1 min repeated for 40 cycles; and a final step at $72^{\circ} \mathrm{C}$ for $10 \mathrm{~min}$ to stop the reaction. Levels of DNase I, caspase-3 and Bcl-2 mRNA were subsequently normalized to $\beta$-actin mRNA levels.

Western blot analysis. Cells were lysed in lysis buffer [50 mM Tris, pH 7.5, $250 \mathrm{mM} \mathrm{NaCl}, 0.1 \%$ sodium dodecyl sulfate (SDS), 2 mM dithiothreitol, 0.5\% NP-40, 1 mM phenylmethanesulfonyl fluoride and protease inhibitor cocktail] on ice for $30 \mathrm{~min}$. Protein fractions were collected by centrifugation at $15,000 \mathrm{x} \mathrm{g}$ at $4^{\circ} \mathrm{C}$ for $10 \mathrm{~min}$ and then were subjected to $10 \%$ SDS-polyacrylimide gel (Sinopharm Chemical Reagent) electrophoresis and were transferred to polyvinylidene difluoride membranes (Merck Millipore, Billerica, MA, USA). The membranes were blocked with $5 \%$ bovine serum albumin (BSA) and incubated with the specific antibodies overnight. The following antibodies were used: Rabbit anti-DNase I (1:500; sc-30058; Santa Cruz Biotechnology, Inc.), mouse anti-caspase-3 (sc-56055; Santa Cruz, USA) and mouse $\beta$-actin (1:5,000; DKM9001; Tianjin Sungene Biotech Co., Ltd., Tianjin, China) to examine the concentrations of DNase I, caspase- 3 and $\beta$-actin proteins in the lysates, respectively. A goat anti-mouse (H+L: 115-035-003)and goat anti-rabbit (H+L: 111-035-003) IgG horseradish peroxidase-labeled secondary antibody (Jackson ImmunoResearch Laboratories, West Grove, PA, USA). was added and visualized using a chemiluminescence reagent (ECL; Engreen Biosystem, Ltd., Beijing, China).

Flow cytometry analysis. The cell apoptotic rate was detected by flow cytometry. Briefly, INS-1 cells were plated in 6-well plates at a density of $5 \times 10^{4}$ cells per well and were incubated at $37^{\circ} \mathrm{C}$ for $24 \mathrm{~h}$. The media was then replaced, so that three groups of cells were present: Cells exposed to constant normal glucose $(11.1 \mathrm{mM})$, constant high glucose $(30 \mathrm{mM})$ and high glucose with DNase I knockout. Subsequent to incubation for an additional $48 \mathrm{~h}$, the cell apoptotic rate was detected by Annexin V staining [Fluorescein Isothiocyanate (FITC) Annexin V Apoptosis Detection kit; BD Biosciences, Franklin Lakes, NJ, USA]. Subsequently, cells were treated with trypsin-ethylenediaminetetraacetic acid and were centrifuged at $1,400 \mathrm{x} g$ for $5 \mathrm{~min}$ at $4^{\circ} \mathrm{C}$. Cells were washed with PBS 2 times, then were resuspended with $100 \mu \mathrm{l}$ binding buffer. Supernatants were then added with $5 \mu \mathrm{l}$ Annexin V-FITC and $5 \mu \mathrm{l}$ propidium iodide. Subsequent to incubation for $15 \mathrm{~min}$ in the dark, $400 \mu \mathrm{l}$ binding buffer was added, and the cells were analyzed by the FACSCanto II flow cytometer (BD Biosciences).

Cell proliferation assays. Cell viability was determined by the Cell Counting Kit-8 assay (Dojindo Molecular Technologies, Inc., Kumamoto, Japan) according to the manufacturer's protocol. Cells $\left(1.5 \times 10^{4}\right)$ were grown as triplicates in 96-well plates and allowed to adhere for $24 \mathrm{~h}$. Cells were exposed to various DNase I concentrations $(1,5,10,50$ and $100 \mathrm{mU} / \mu \mathrm{l})$, for $24 \mathrm{~h}$, then $10 \mu \mathrm{l}$ tetrazolium substrate was added to each well of the plate. Plates were incubated at $37^{\circ} \mathrm{C}$ for $1 \mathrm{~h}$, then the optical density was measured at $450 \mathrm{~nm}$ using a Bio-Rad 680 Enzyme-linked Immunosorbent Assay Reader (Bio-Rad Laboratories, Inc., Hercules, CA, USA). Cell viability was expressed as a percentage of control cells (non-treated).

Terminal deoxynucleotidyl transferase dUTP nick-end labeling (TUNEL) assay. For the TUNEL assay, INS-1 cells were seeded into 96-well plates $\left(2 \times 10^{4}\right.$ cells/well $)$ and treated with or without high glucose for $12 \mathrm{~h}$. Subsequently, the cells were incubated with 50 or $100 \mathrm{mM}$ DNase I in RPMI-1640 containing $10 \%$ FCS for $24 \mathrm{~h}$. The cells were then washed with PBS (HyClone, Logan, UT, USA), fixed in 4\% paraformaldehyde (Sinopharm Chemical Reagent), and permeabilized with $0.1 \%$ Triton X-100 (Sigma-Aldrich) in PBS/BSA solution. The TUNEL assay was performed using the In Situ Cell Death Detection kits (Roche Diagnostics, Indianapolis, IN, USA). 
A

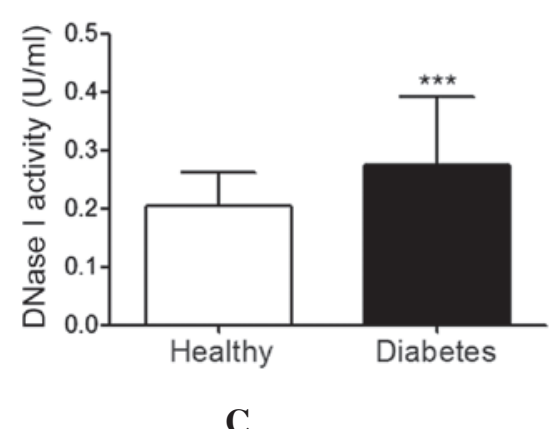

B

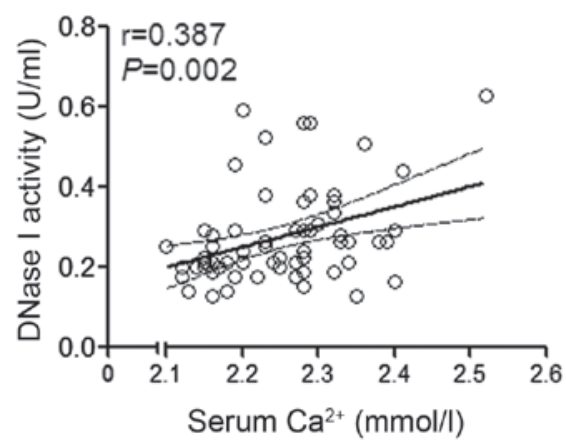

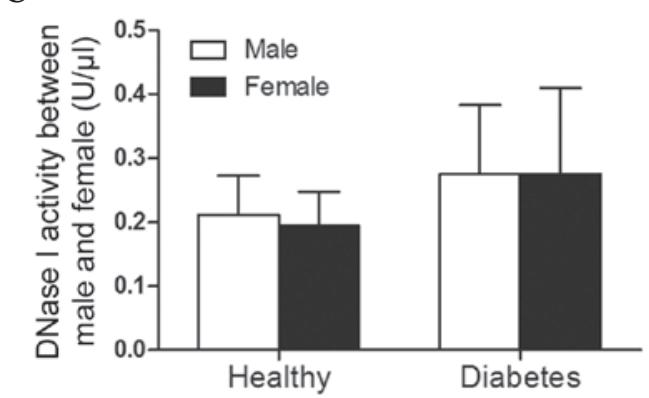

Figure 1. DNase I activity in human serum. (A) DNase I activity in human serum. (B) The correlation of DNase I activity with calcium. (C) DNase I activity Spearman correlation coefficient. All data are presented as the mean \pm standard deviation. ${ }^{* * *}$ P $<0.001$. DNase I, deoxyribonuclease I.

Statistical analyses. SPSS software, version 11.0 (SPSS, Inc., Chicago, IL, USA) was used for the statistical analysis. Statistical analyses for parametric data and nonparametric data were performed using the Kolmogorov-Smirnov test and the Mann-Whitney U test, respectively. Spearman single regression analyses were used to assess the correlation between healthy controls and patients with diabetes. All of the data are presented as the mean \pm standard deviation. $\mathrm{P}<0.05$ was considered to indicate a statistically significant difference.

\section{Results}

Human DNase I activity. A total of 66 patients with diabetes were enrolled in the current study. Of the participants, 22 were women $(33.3 \%)$ and 44 were men $(66.7 \%)$ with a mean age of $52.68 \pm 8.91$ (range, $35-65$ years). Of the 52 healthy controls, 24 were women $(46.1 \%)$ and 28 were men $(53.8 \%)$, with a mean age of 50.47 \pm 8.37 (range, 35-65 years). Compared with the healthy controls, DNase I activity was observed to tbe significantly elevated in patients with type 2 diabetes $(0.2 \pm 0.06 \mathrm{U} / \mathrm{ml}$ vs. $0.27 \pm 0.12 \mathrm{U} / \mathrm{ml} ; \mathrm{P}<0.001 ;$ Fig. $1 \mathrm{~A})$. In addition, this increase was identified to be positively correlated with serum $\mathrm{Ca}^{2+}$ concentration ( $\mathrm{r}=0.387$; $\mathrm{P}=0.002$; Fig. 1B), however no correlation of DNase I activity with HbAlc $(r=0.06 ; \mathrm{P}=0.641)$ or fasting glucose $(\mathrm{r}=0.132 ; \mathrm{P}=0.297)$ was observed. No significant difference of the DNase I activity was identified between male and female patients with type 2 diabetes (Fig. 1C).

Immunohistochemistry results demonstrated that islets in the diabetic patients exhibited an irregular morphology, with poor insulin staining and increased glucagon staining. The DNase I expression was marginally increased when compared with those without diabetes (Fig. 2).
DNase I gene expression was increased in high glucose-cultured cells. Compared with the normal group, DNase I was significantly increased in the $30 \mathrm{mM}$ glucose group, which is in agreement with a previous study (10). High glucose additionally increased the caspase- 3 expression, whereas this increase was reversed by DNase I siRNA transfection (Fig. 3B and C). Notably, it was observed that when DNase I was knocked down by DNase I siRNA, the caspase-3 level was observed to be reduced when compared with the high glucose group. The specific mechanism of this remains unclear. In the current study, it was also identified that high levels of glucose resulted in cell apoptosis. Subsequent to transfection with siRNA for $48 \mathrm{~h}$, the rate of cell apoptosis was examined, and it was identified hat the apoptotic rate in the normal, high glucose and high glucose with siRNA groups were 7.1\%, $18.1 \%$ and $9.9 \%$, respectively (Fig. 3D).

High glucose with $50 \mathrm{mU} / \mu \mathrm{l}$ DNase I leads to cell apoptosis. To further explain the specific effects of DNase I in cell proliferation, cells were cultured with high glucose and DNase I siRNA. The cell proliferation results indicated that $50 \mathrm{mU} / \mu 1$ DNase I greatly suppressed the cell proliferation $(\mathrm{P}<0.05$; Fig. $4 \mathrm{~A})$. To more accurately simulate the internal environment of patients with type 2 diabetes, the cells were cultured with high glucose and $50 \mathrm{mU} / \mu \mathrm{l}$ DNase I, and it was identified that this resulted in a significant increase in caspase-3 levels (Fig. 4B and C). The TUNEL results indicated that $50 \mathrm{mU} / \mu \mathrm{l}$ DNase I with high glucose resulted in a marked increase in cell apoptosis, when compared with the high glucose-treatment alone group. The flow cytometry results were in agreement with those from TUNEL, indicating that high glucose combined with $50 \mathrm{mU} / \mu \mathrm{l}$ DNase I resulted in clear cellular apoptosis (34.6\%), when compared with the normal and control groups $(6.9 \%$ and $17.3 \%$, respectively) (Fig. 4D). 

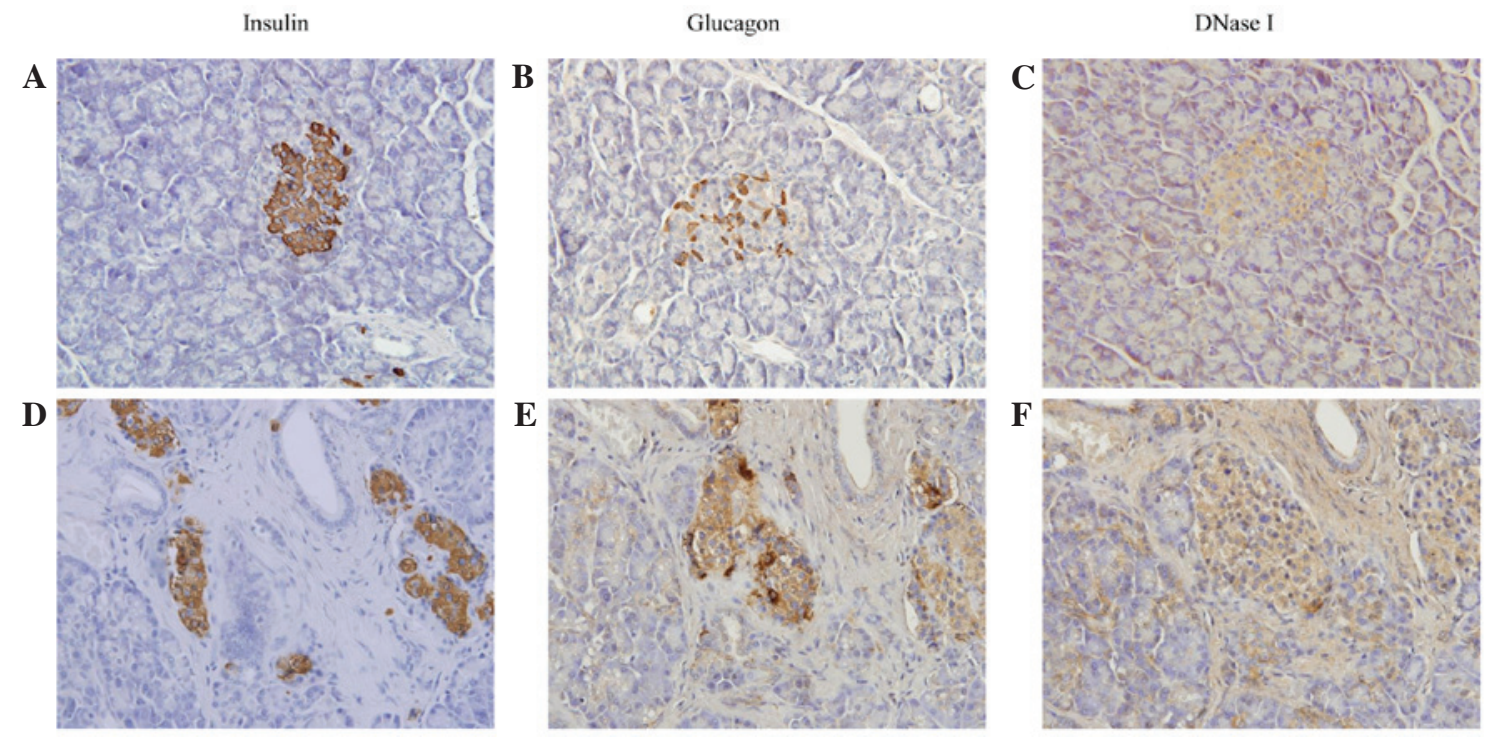

Figure 2. Immunohistochemistry of the human pancreas. Pancreatic tissues from patients with pancreatic cancer, with or without type 2 diabetes were stained with insulin, glucagon, and DNase I. (A) Insulin, (B) glucagon and (C) DNase I staining of tissues from patients without type 2 diabetes. (D) Insulin, (E) glucagon and (F) DNase I staining of tissues from patients with type 2 diabetes. DNase I, deoxyribonuclease I.

A

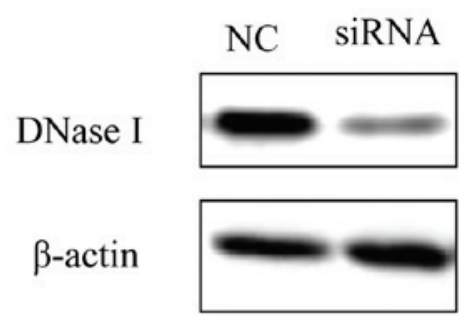

C

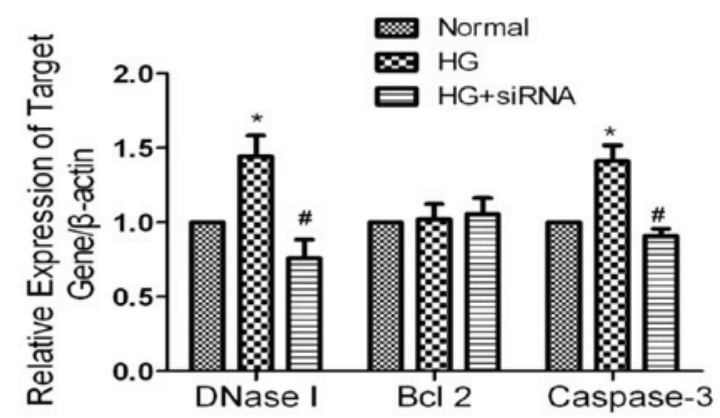

D

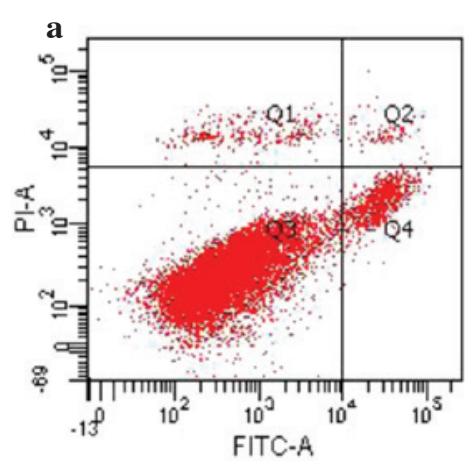

B

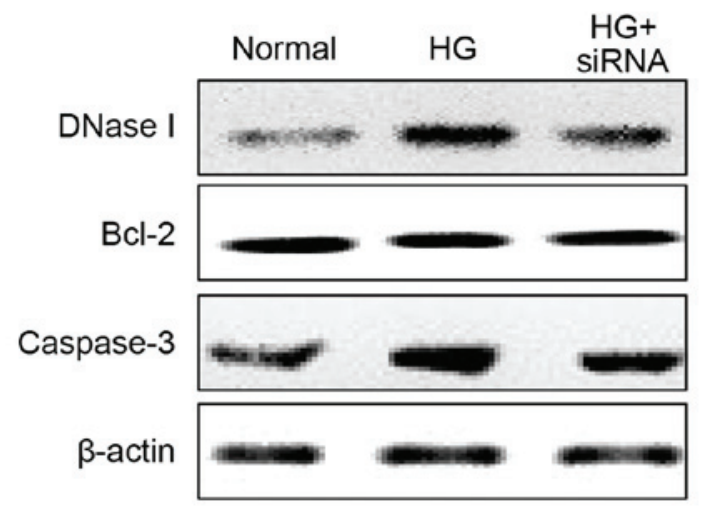

b

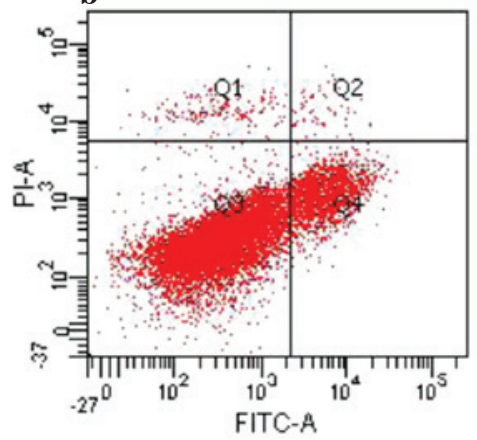

c

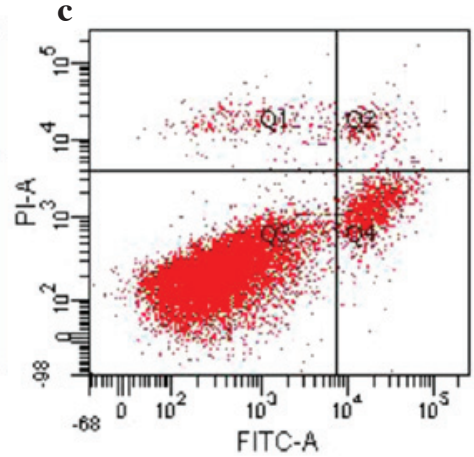

Figure 3. DNase I knockdown can reduce the apoptosis of cells cultured with high glucose. (A) Knockdown efficiency examined by western blotting. Expression of DNase I, Bcl-2 and caspase-3 in the three groups were examined by (B) western blotting and (C) reverse transcription-quantitative polymerase chain reaction. (D) Apoptotic rate examined by flow cytometry [(a), normal; (b), high glucose; and (c), siRNA group]. Data are expressed as the mean \pm standard deviation from three independent experiments. ${ }^{*} \mathrm{P}<0.05$ vs. the normal group and ${ }^{\#} \mathrm{P}<0.05$ vs. the control group. DNase I, deoxyribonuclease I; siRNA, small interfering RNA; NC, negative control; N, normal; HG, high glucose; PI, propidium iodide; FITC, fluorescein isothiocyanate. 
A

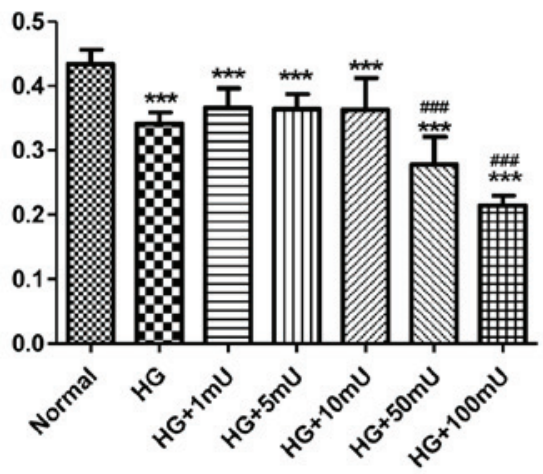

B

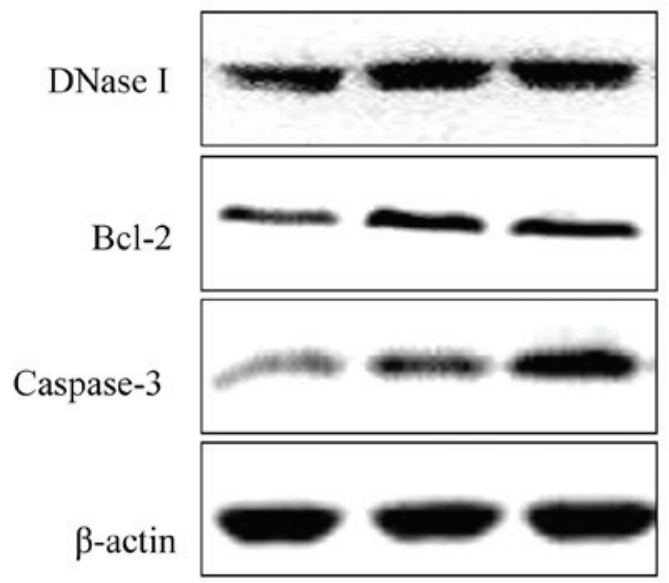

C

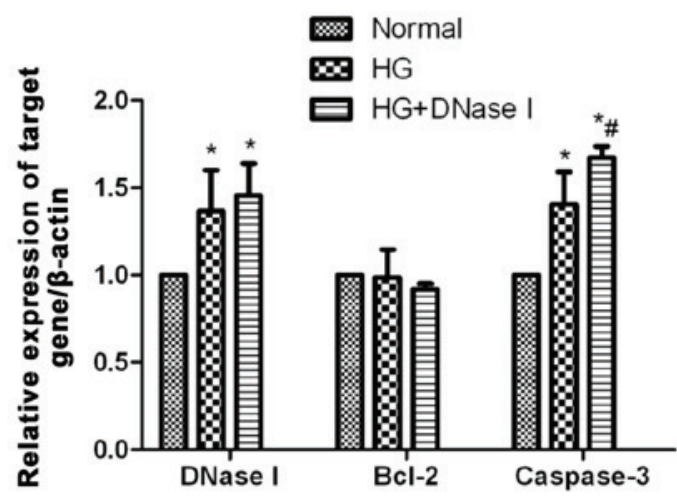

D $\mathbf{a}$
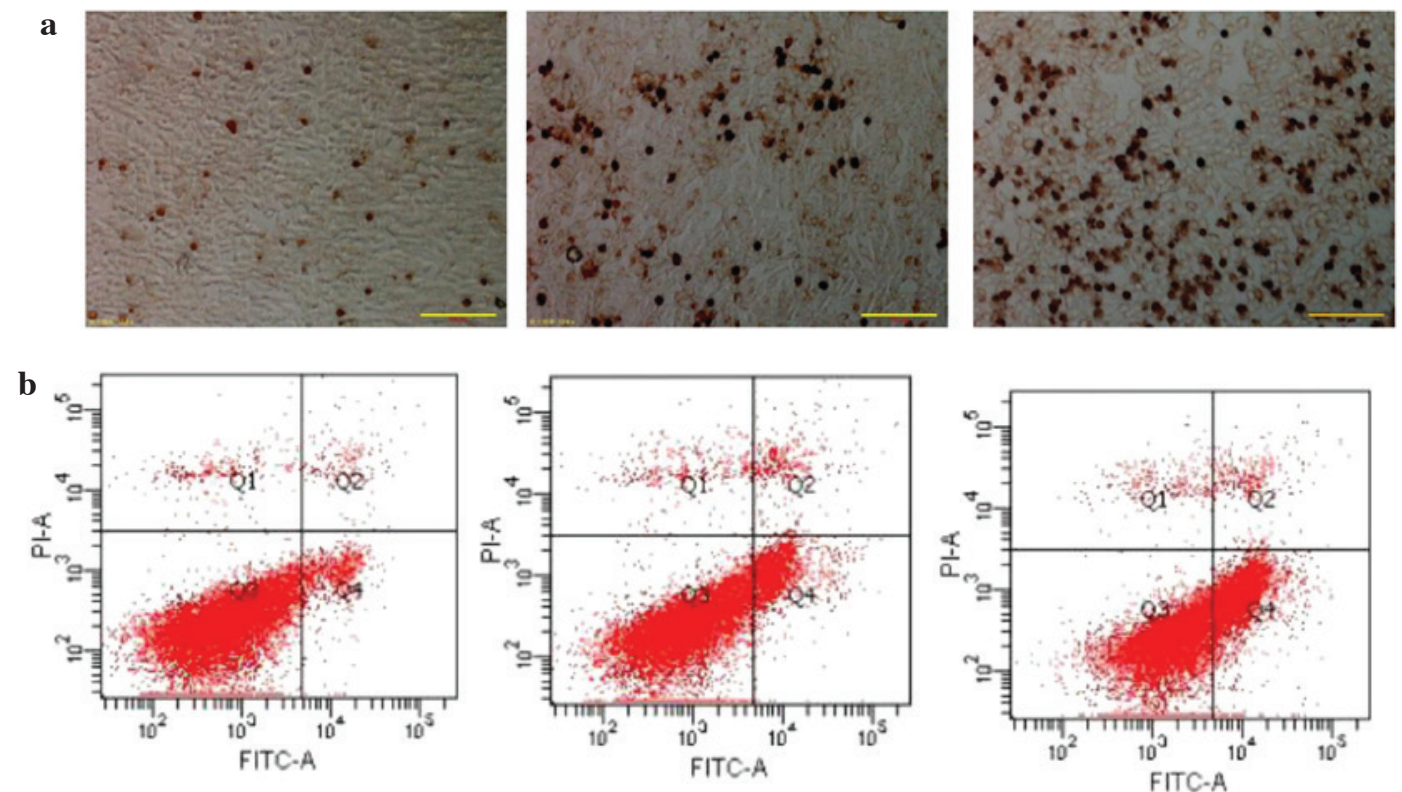

Figure 4. DNase I combined with high glucose induced cell apoptosis. (A) Cell viability assessed by the Cell Counting Kit-8 assay. The expression levels of DNase I, Bcl-2 and caspase-3 in the three groups were examined by (B) western blotting and (C) reverse transcription-quantitative polymerase chain reaction. (D) Cell apoptosis results from (a) the terminal deoxynucleotidyl transferase dUTP nick-end labeling assay and (b) flow cytometry. Data are expressed as the mean \pm standard deviation from three independent experiments. ${ }^{*} \mathrm{P}<0.05$ and ${ }^{* * *} \mathrm{P}<0.001$ vs. the normal group and ${ }^{\#} \mathrm{P}<0.05$ and ${ }^{\# \# \#} \mathrm{P}<0.001$ vs. the control group. DNase I, deoxyribonuclease I; HG, high glucose; PI, propidium iodide; FITC, fluorescein isothiocyanate.

\section{Discussion}

Type 2 diabetes mellitus is a disease of high prevalence, however its etiology remains to be fully elucidated. Numerous factors including $\beta$ cell apoptosis, oxidative stress and inflammation have been suggested to contribute to the morbidity of this disease. However, the specific mechanisms remain unclear. The role of DNase I in SEL and AMI has been previously investigated, however at present to the best of our knowledge, no correlative studies on its association with type 2 diabetes have been conducted $(6,11,12)$. In the current study, it was identified that DNase I levels were increased in type 2 diabetes, 
and this increase in combination with high glucose may aggravate $\beta$ cell apoptosis. To the best of our knowledge, the current study is the first to suggest that DNase I may be involved in the pathophysiology of type 2 diabetes in the development of metabolic syndrome.

DNase I is ubiquitously expressed in mammalian tissues, particularly in the pancreas (13). It has been suggested to be highly cytotoxic in mammalian cells when its activity and expression is increased, as it can effectively digest single- and double-stranded DNA (14). In addition, as a 'waste-management enzyme', it can trigger apoptosis $(15,16)$. Since its discovery in the bovine pancreas in 1905, correlations have only been identified between DNase I and SLE and AMI (17). In SLE, the reduction of DNase I levels were considered to be the predominant cause of antigen accumulation, leading to autoimmunity (18). In AMI, the enzymatic activity was identified to be elevated within $3 \mathrm{~h}$ and returned to basal levels within $24 \mathrm{~h}$, thus it is used for the early diagnosis of unstable angina pectoris or non-ST-segment elevation myocardial infarction $(19,20)$. The associations between activity of this enzyme and type 2 diabetes require elucidation.

In the present study, it was identified that high glucose was able to induce an increase in DNase I expression, which resulted in cell apoptosis. In order to confirm that apoptosis was correlated with DNase I expression, siRNA was used to knock down DNase I. It was observed that a reduction in DNase I expression resulted in significant reductions in apoptotic rate and caspase-3 protein levels, even in the presence of high glucose. To further investigate the role of DNase I in cell apoptosis, cells were co-cultured with high glucose and $50 \mathrm{mU} / \mu \mathrm{l}$ DNase I to simulate the conditions of type 2 diabetes. Notably, a significant increase in apoptosis was observed in cells with $50 \mathrm{mU} / \mu 1$ DNase I and high glucose, compared with high glucose-culture alone. This suggested that DNase I may participate in $\beta$ cell apoptosis in type 2 diabetes.

However, the specific mechanisms of DNase I in type 2 diabetes remain to be fully elucidated, due to the complex interactions among various factors. DNase I hypersensitive sites (DHSs; short regions of chromatin that are highly sensitive to DNase I digestion) in the cis-regulatory region on chromatin has been previously suggested to be correlated with disease $(21,22)$. Human regulatory DNA encompasses a variety of $c i s$-regulatory elements, within which, the cooperative binding of transcription factors creates focal alterations in chromatin structure. Maurano et al (23) identified that disease-associated variants in DHSs systematically alter the transcription factor recognition sequences and allelic chromatin states, and form regulatory networks. Stitzel et al (24) reported that DNase I hypersensitive sites in the cis-regulatory elements of islet $\beta$-cells are increased in the pancreatic islets, which may contribute to the morbidity of type 2 diabetes. Thus, it is suggested that in type 2 diabetes, the increase of DNase I may affect the short regions of chromatin cleavage and influence the secretion of insulin. This may result in the development of type 2 diabetes.

In summary, the present study provided novel insight into the central role of DNase I in high glucose-induced pancreatic $\beta$-cell apoptosis. It was demonstrated that high glucose was able to increase the expression of DNase I, and that this may aggravate $\beta$-cell apoptosis. These observations may prove useful for the development of novel new therapeutic strategies for the treatment of type 2 diabetes mellitus.

\section{Acknowledgements}

The authors would like to thank Dr Shi Rui Li for their technical assistance in conducting the experiments. The current study was supported by grants from the National Natural Scientific Foundation of China (grant nos. 81173422 and 81402814).

\section{References}

1. Butler AE, Janson J, Bonner-Weir S, Ritzel R, Rizza RA and Butler PC: Beta-cell deficit and increased beta-cell apoptosis in humans with type 2 diabetes. Diabetes 52: 102-110, 2003.

2. Mandrup-Poulsen T: Apoptotic signal transduction pathways in diabetes. Biochem Pharmacol 66: 1433-1440, 2003.

3. Ardestani A, Paroni F, Azizi Z, Kaur S, Khobragade V, Yuan T, Frogne T, Tao W, Oberholzer J, Pattou F, et al: MST1 is a key regulator of beta cell apoptosis and dysfunction in diabetes. Nat Med 20: 385-397, 2014.

4. Zhou Z, Zhu C, Ren J and Dong S: A graphene-based real-time fluorescent assay of deoxyribonuclease I activity and inhibition. Anal Chim Acta 740: 88-92, 2012.

5. Kaneko Y, Takeshita H, Mogi K, Nakajima T, Yasuda T, Itoi M, Kuwano H and Kishi K: Molecular, biochemical and immunological analyses of canine pancreatic DNase I. J Biochem 134: 711-718, 2003.

6. Funakoshi A, Wakasugi $\mathrm{H}$ and Ibayashi $\mathrm{H}$ : Clinical investigation of serum deoxyribonuclease: II. Clinical studies of serum deoxyribonuclease activity in pancreatic disease. Gastroenterol Jpn 14: 436-440, 1979.

7. Martinez-Valle F, Balada E, Ordi-Ros J, Bujan-Rivas S, Sellas-Fernandez A and Vilardell-Tarres M: DNase 1 activity in patients with systemic lupus erythematosus: Relationship with epidemiological, clinical, immunological and therapeutical features. Lupus 18: 418-423, 2009.

8. Kuribara J, Tada H, Kawai Y, Kawaguchi R, Hoshizaki H, Arakawa K, Kitayama M, Kajinami K, Kurabayashi M, Oshima S, et al: Levels of serum deoxyribonuclease I activity on admission in patients with acute myocardial infarction can be useful in predicting left ventricular enlargement due to remodeling. J Cardiol 53: 196-203, 2009.

9. St-Onge L, Wehr R and Gruss P: Pancreas development and diabetes. Curr Opin Genet Dev 9: 295-300, 1999.

10. Zhu B, Gong Y, Chen P, Zhang H, Zhao T and Li P: Increased DNase I activity in diabetes might be associated with injury of pancreas. Mol Cell Biochem 393: 23-32, 2014.

11. Tinazzi E, Puccetti A, Gerli R, Rigo A, Migliorini P, Simeoni S, Beri R, Dolcino M, Martinelli N, Corrocher R and Lunardi C: Serum DNase I, soluble Fas/FasL levels and cell surface Fas expression in patients with SLE: A possible explanation for the lack of efficacy of hrDNase I treatment. Int Immunol 21: 237-243, 2009.

12. Errami Y, Naura AS, Kim H, Ju J, Suzuki Y, El-Bahrawy AH, Ghonim MA, Hemeida RA, Mansy MS, Zhang J, et al: Apoptotic DNA fragmentation may be a cooperative activity between caspase-activated deoxyribonuclease and the poly(ADP-ribose) polymerase-regulated DNAS1L3, an endoplasmic reticulum-localized endonuclease that translocates to the nucleus during apoptosis. J Biol Chem 288: 3460-3468, 2013.

13. Shiokawa D and Tanuma S: Characterization of human DNase I family endonucleases and activation of DNase gamma during apoptosis. Biochemistry 40: 143-152, 2001.

14. Rosner K, Kasprzak MF, Horenstein AC, Thurston HL, Abrams J, Kerwin LY, Mehregan DA and Mehregan DR: Engineering a waste management enzyme to overcome cancer resistance to apoptosis: Adding DNase1 to the anti-cancer toolbox. Cancer Gene Ther 18: 346-357, 2011.

15. Oliveri M, Daga A, Cantoni C, Lunardi C, Millo R and Puccetti A: DNase I mediates internucleosomal DNA degradation in human cells undergoing drug-induced apoptosis. Eur J Immunol 31: 743-751, 2001.

16. Hall AK: Molecular interactions between G-actin, DNase I and the beta-thymosins in apoptosis: A hypothesis. Med Hypotheses 43: 125-131, 1994. 
17. Martínez Valle F, Balada E, Ordi-Ros J and Vilardell-Tarres M: DNase 1 and systemic lupus erythematosus. Autoimmun Rev 7: 359-363, 2008

18. Martinez-Valle F, Balada E, Ordi-Ros J, Bujan-Rivas S, Sellas-Fernandez A and Vilardell-Tarres M: DNase1 activity in systemic lupus erythematosus patients with and without nephropathy. Rheumatol Int 30: 1601-1604, 2010.

19. Fujibayashi K, Kawai Y, Kitayama M, Akao H, Ishida R Motoyama A, Wakasa M, Arakawa K, Ueki M, Kajinami K and Yasuda T: Serum deoxyribonuclease I activity can be a useful diagnostic marker for the early diagnosis of unstable angina pectoris or non-ST-segment elevation myocardial infarction. J Cardiol 59: 258-265, 2012.

20. Yasuda T, Iida R, Kawai Y, Nakajima T, Kominato Y, Fujihara J and Takeshita H: Serum deoxyribonuclease I can be used as a useful marker for diagnosis of death due to ischemic heart disease. Leg Med (Tokyo) 11 (Suppl 1): S213-S215, 2009.
21. Boyle AP, Davis S, Shulha HP, Meltzer P, Margulies EH, Weng Z, Furey TS and Crawford GE: High-resolution mapping and characterization of open chromatin across the genome. Cell 132: 311-322, 2008.

22. Thurman RE, Rynes E, Humbert R, Vierstra J, Maurano MT, Haugen E, Sheffield NC, Stergachis AB, Wang H, Vernot B, et al: The accessible chromatin landscape of the human genome. Nature 489: 75-82, 2012.

23. Maurano MT, Humbert R, Rynes E, Thurman RE, Haugen E, Wang H, Reynolds AP, Sandstrom R, Qu H, Brody J, et al: Systematic localization of common disease-associated variation in regulatory DNA. Science 337: 1190-1195, 2012.

24. Stitzel ML, Sethupathy P, Pearson DS, Chines PS, Song L, Erdos MR, Welch R, Parker SC, Boyle AP, Scott LJ, et al: Global epigenomic analysis of primary human pancreatic islets provides insights into type 2 diabetes susceptibility loci. Cell Metab 12: 443-455, 2010. 